\title{
Real-time screening of biocatalysts in live bacterial colonies
}

\author{
Cunyu Yan, ${ }^{\text {a }}$ Fabio Parmeggiani, ${ }^{\text {a }}$ Emrys A. Jones, ${ }^{\mathrm{b}}$ Emmanuelle Claude, ${ }^{\mathrm{b}}$ Shaneela A. Hussain, ${ }^{\mathrm{a}}$ Nicho- \\ las J. Turner, ${ }^{\text {a }}$ Sabine L. Flitsch ${ }^{a *}$ and Perdita E. Barran ${ }^{\mathrm{a} *}$ \\ ${ }^{\text {a }}$ Manchester Synthetic Biology Research Centre for Fine and Speciality Chemicals (SYNBIOCHEM), Manchester Institute \\ of Biotechnology, School of Chemistry, The University of Manchester, 131 Princess Street, M1 7DN, Manchester, United \\ Kingdom. \\ ${ }^{\mathrm{b}}$ Waters Corp., Stamford Avenue, Altrincham Road, SK9 4AX, Wilmslow, United Kingdom.
}

\begin{abstract}
Screening of bacterial colonies to identify new biocatalytic activities is a widely adopted tool in biotechnology, but is constrained by the requirements for colorimetric or tagbased detection methods. Herein we report a label-free screening platform for biotransformations in live colonies using desorption electrospray ionization coupled with ion mobility mass spectrometry imaging (DiBT-IMMS). The screening method is demonstrated for both ammonia lyases and P450 monooxygenases expressed within live bacterial colonies and is shown to enable multiplexing of enzyme variants and substrate libraries simultaneously.
\end{abstract}

One of the most simple and widely used methods in the identification of new biocatalysts is the expression of gene variants within bacterial colonies grown on media such as agar plates. However, the need to screen large numbers of such colonies for enhanced activity often constitutes the major bottleneck in the identification of new biocatalytic activities. ${ }^{1}$ Colorimetric screening methods have been developed to identify target protein expression and activity, but the prerequisite of finding suitable chromophores inevitably places a limit on the type of chemical transformation that can be detected. ${ }^{2}$ Furthermore, these methods are generally hampered by a high incidence of false positive/negative results and difficulties with precise quantification.

Mass spectrometry (MS) with its unique combination of specificity, sensitivity and speed (the "three S" advantages cited by McLafferty $^{3}$ ) is an obvious analytical route for screening biotransformation reactions. It also has been successfully applied to directed evolution assays ${ }^{4}$ for example in determination of the enantioselectivity of enzyme variants. ${ }^{5}$ This said, MS screening from biotransformations in vitro or in colony necessitates multiple liquid handling steps including extraction and subsequent removal of the organic phase along with some form of chromatographic separation, to best analyse the reaction mixture, which can work against high throughput. Ambient ionisation methodologies coupled with MS, and in particular desorption electrospray ionization (DESI) introduced by Cooks et al., ${ }^{6,7}$ circumvent some of these hurdles, by sampling directly from an analytical target at room temperature, in real time,$^{8}$ but to date have not been applied to screen biotransformation reactions.

Amongst many notable advantages to ambient ionization MS during the past decade is its combination with MS based imaging to probe chemical species from surfaces as a function of spatial distribution. ${ }^{9}$ Several multidisciplinary applications for imaging MS techniques have been developed ${ }^{10}$, including in clinical chemistry (e.g., analysis of blood samples) ${ }^{11}$ and in microbiology (e.g., microorganism characterisation by metabolic profiling). ${ }^{12}$ To the best of our knowledge, the application of DESI methods to highthroughput screening of biocatalysis directly from bacterial colonies on agar plates has not previously been described. Given the ease of analysis directly from biological material under ambient conditions, in situ DESI-MS is highly attractive as a screening method for biocatalysts, since post analysis/on hit discovery DNA can then be recovered, amplified and sequenced after mass analysis, thus directly linking genotype with phenotype. Here we report a label-free on-colony screening method (Figure 1) based on ambient ionisation coupled with imaging MS, integrating the following three components provided by off-the-shelf instrumentation: (i) DESI to ionise substrates and reaction products from the biotransformation; (ii) ion mobility (IM) spectrometry to separation the plethora of ions produced following DESI from live colonies, which dramatically reduces background chemical noise, ${ }^{13}$ (iii) imaging MS to locate the colony on the plate from distinctive lipid signals, and to identify specific product species from successful biotransformations. This DESI-IMMS analysis allows multiplexed analysis of substrate libraries in a single experiment,

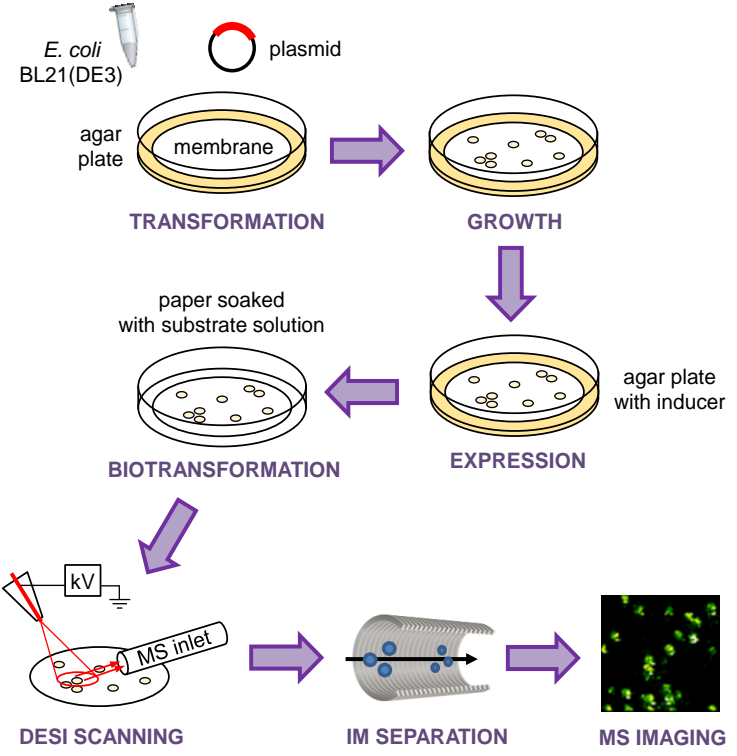

Figure 1. Workflow of DiBT-IMMS imaging of bacterial colonies expressing biocatalysts on agar plates under ambient conditions. 
and removes the need for sample preparation enabling products catalysed in live bacterial colonies to be analysed directly. We term this on colony method Direct BioTransformation IMMS (DiBT-IMMS).

The basic workflow of our DiBT-IMMS screening platform as shown in Figure 1 has been worked up from previous colonybased screens that were developed in our laboratory and by others for colorimetric assays. ${ }^{1,2} \mathrm{E}$. coli cells harbouring the desired plasmid/library are plated onto a nylon membrane laid on an agar plate. After incubation, the membrane is transferred to another plate containing the inducer for expression. The membrane is then incubated on filter paper soaked with substrate solution and subsequently submitted to DESI-IM-MS analysis, tracking the product(s) of the biotransformation.

The surface of the membrane is swept line-by-line (100 $\mu \mathrm{m}$ line width) with a spray of ionised $\mathrm{MeOH} / \mathrm{H}_{2} \mathrm{O}$ droplets and the resulting ions are continuously extracted into the mass spectrometer. By correlating the intensity of the desired signal $(\mathrm{m} / \mathrm{z}$ corresponding to the specific product) to the $x-y$ physical position on the $2 \mathrm{D}$ surface of the plate, the data can be processed as a mass-selected image representing visually the areas with higher product concentration. The presence of any product can be imaged by extracting the whole data set with the predicted $\mathrm{m} / \mathrm{z}$. At the same time, the physical position of each of the colonies on the surface can be monitored by tracking an abundant common species (here the C18:1 fatty acid, $m / z, 281$ ).

Initially, both the feasibility and sensitivity of DiBT-IMMS were tested using a solution of L-phenylalanine as a standard, which was detected when spotted onto an untreated nylon membrane with a limit of detection per unit area of $>50 \mathrm{nmol} \mathrm{mm}-2$ (Figure 2a).

As a first application of DiBT-IMMS to a whole-cell biocatalytic reaction, we chose to monitor the asymmetric addition of ammonia to cinnamic acid 1a (Figure $2 \mathrm{~b}$ ) catalysed by phenylalanine ammonia lyase (PAL). ${ }^{14}$ This reaction is a synthetically useful transformation with no known chemical counterpart, and presents a challenge for screening due to the lack of easily accessible colorimetric assays, which made it an ideal candidate for a labelfree approach. A membrane carrying colonies of E. coli cells producing active PAL (AvPAL from Anabaena variabilis) ${ }^{15}$ was incubated with a solution of $1 \mathbf{a}$ in ammonium carbonate buffer. The position of all bacterial colonies could be imaged using the reference signal from generic C18:1 cell wall lipid $(\mathrm{m} / \mathrm{z}, 281)$, which showed little change over the incubation period (Figure $2 \mathrm{c}$, top panels in blue). In contrast, mass selective images of $2 \mathbf{a}(\mathrm{m} / \mathrm{z} .164$, Figure 2c, bottom panels in green) were only observed following biotransformation. Data from the same membrane over several hours allowed us to observe an increase in signal for 2a (Figure $2 c$ ), indicating that quantitative determination of product formation is possible.

An important challenge in the development of useful biocatalysts is to expand the substrate range beyond natural compounds to non-natural analogues. Here, the DiBT-IMMS method performs well as demonstrated by testing non-natural analogues $\mathbf{1 b}-\mathbf{j}$ of cinnamic acid in the whole-cell system (Figure 3a). All products $\mathbf{2 b}$-j resulting from the PAL reaction were clearly visible when imaging colonies expressing PAL (Figure $3 b$ and Supplementary Figure S1a). The results were validated by HPLC methods (see Supporting Information for conditions and representative traces).

With non-natural substrates $\mathbf{1 b}$-j there was also the opportunity to exploit natural isotope ratios to increase signal in the detection of any given analyte in the complex biological matrix. For a
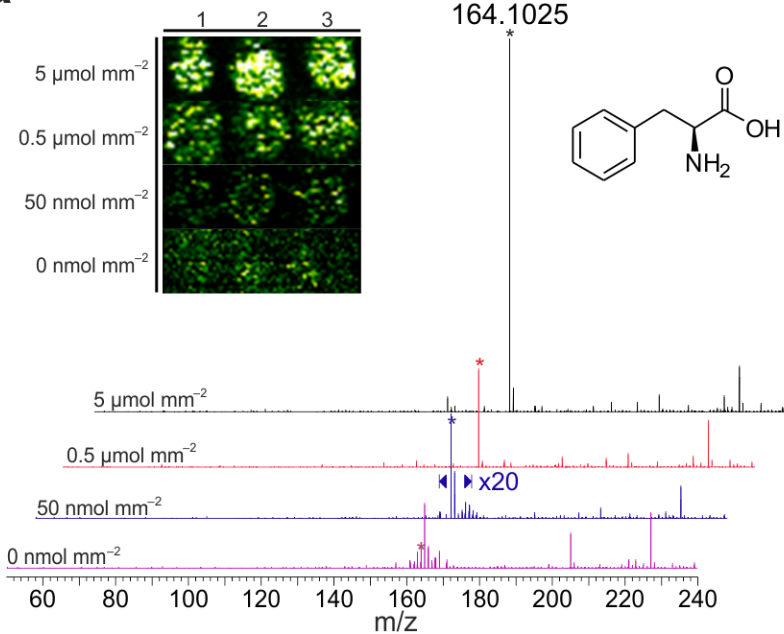

b

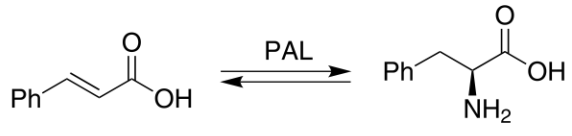

$1 a$

2a

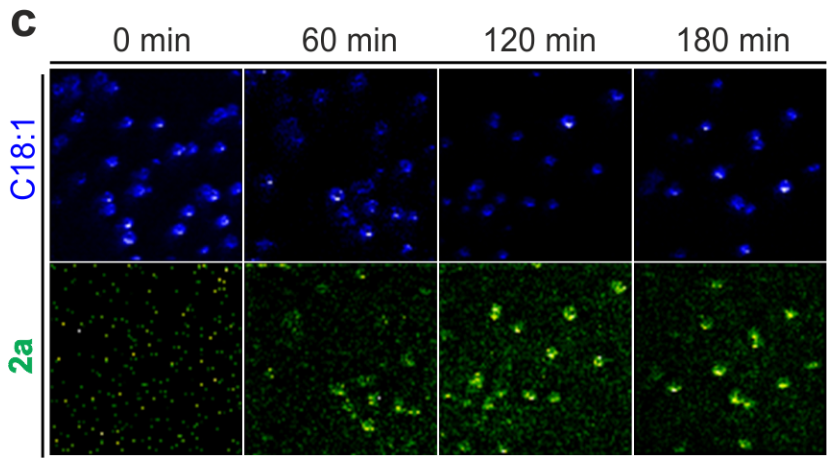

Figure 2. DiBT-IMMS screening of bacterial colonies. (a) Mass spectra illustrating the sensitivity of this technique employing Lphenylalanine as a model target compound (asterisk labelled peak). (b) Model PAL reaction examined here. (c) DiBT-IMMS mass-selected images from colonies expressing PAL, during the incubation with 1a (blue: selection of ion $\mathrm{m} / \mathrm{z}, 281$ assigned to the cell wall lipid C18:1; green: selection of ion $\mathrm{m} / \mathrm{z} 164$ assigned to L-Phe 2a). The four samples were cut from the same membrane and incubated with the substrate for different times.

example, for $\mathbf{2} \mathbf{b}-\mathbf{j}$ the selection of unit masses from the product ion distribution allows detection of overlapping images for isotopically distinct products, which can be used to further validate assignment and increase the sensitivity of the screen (Figure 3b). The outstanding advantage of IM separation in the reduction of chemical background noise is instrumental for the obtainment of high quality spectra (for instance, see Supplementary Figure S2).

As a second example of the DiBT-IMMS screening platform, we chose to study $\mathrm{C}-\mathrm{H}$ activation reactions mediated by $\mathrm{P} 450$ monooxygenases, ${ }^{16}$ a class of enzymes that presents challenges to assay development ${ }^{17}$ and is of greater general interest beyond biocatalysis. ${ }^{18}$ A typical reaction is the $\mathrm{P} 450$-mediated hydroxylation of diclofenac 3, a commonly used nonsteroidal anti-inflammatory drug (Figure 3c) which is of relevance in terms of biocatalysis, toxicology and environmental contamination. ${ }^{19}$ Using the DiBTIMMS workflow as before (Figure1) we were able to demonstrate 
a

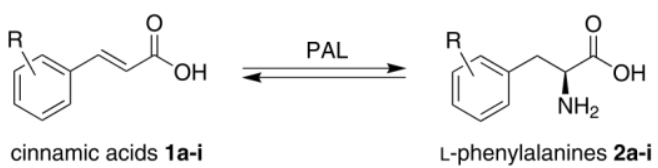

cinnamic acids $1 \mathrm{a}-\mathbf{i}$

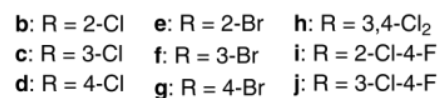

b

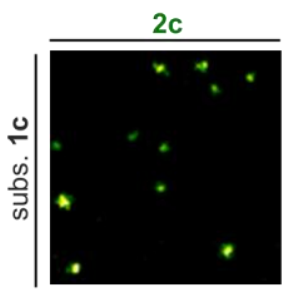

$2 d$

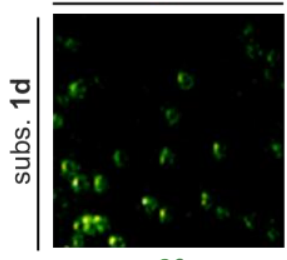

$2 f$

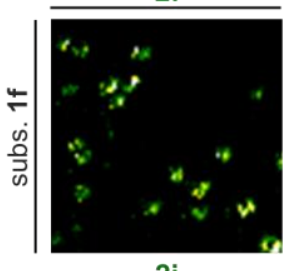

$2 \mathrm{i}$
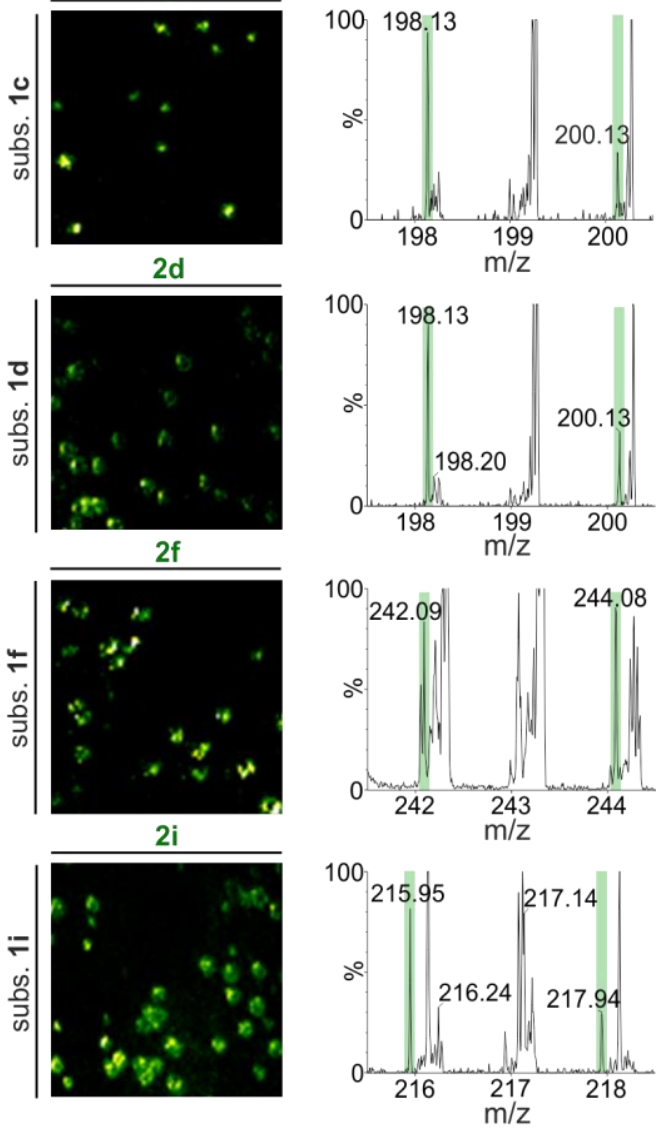

C

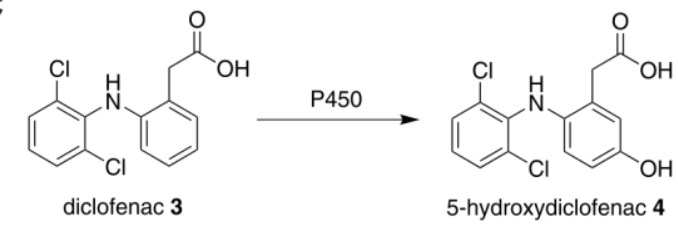

d
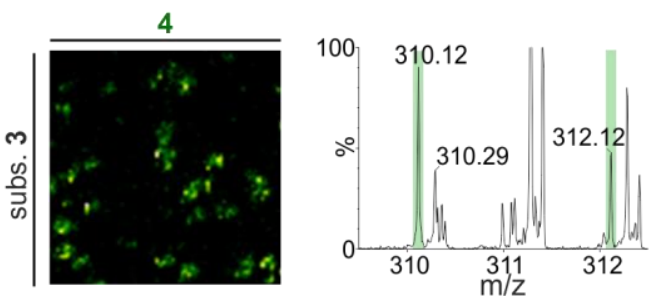

Figure 3. Application of DiBT-IMMS to screen biocatalytic reactions. (a) Expansion of the substrate scope of the PAL reaction shown in Figure 1b. (b) DiBT-IMMS imaging of the PAL reaction with different substrates, performed on colonies producing AvPAL (green: product). (c) Extension to a cofactor-dependent reaction, P450-mediated oxidation of diclofenac. (d) DiBT-IMMS imaging of the $\mathrm{P} 450$ reaction, performed on colonies producing P450-RhF (green: product). the hydroxylation of diclofenac 3 mediated by whole cells expressing $\mathrm{P} 450$ monooxygenase ( $\mathrm{P} 450-\mathrm{RhF}$ from Rhodococcus sp. $)^{20}$ to give 5-hydroxydiclofenac 4 (Figure $3 \mathrm{~d}$ and Supplementary Figure S1b).

To demonstrate the use of DiBT-IMMS as an effective screening tool, the technology was challenged to first identify actives among mixtures of colonies and subsequently to correlate these active phenotypes with genotypes by subsequent DNA extraction and sequencing directly from the agar plates (Figure 4). Mixtures of bacterial colonies producing either active PAL enzyme or empty vector were grown on plates and imaged as before (Figure $4 a, b$ ) both for generic lipid to identify colonies and for PAL product to identify active phenotypes. A significant number of colonies appeared inactive and subsequent DNA analysis showed excellent correlation to product detection (Supplementary Figure S3). This demonstrates the effectiveness of our strategy in the screening of extensive mutant libraries with large differences in activity and/or low success rates, common conditions in enzyme evolution projects. The development of more accurate quantitation procedures is currently ongoing in order to enhance the screening capability of DiBT-IMMS to smaller activity differences.

Finally, the possibility for multiplexing the assay was investigated by screening two different substrates (meta-substituted cinnamic acids 1c and 1f) at the same time (Figure 4c) with excellent overlap of activity profiles. This simultaneous detection of more than one product adds a further dimension to DiBT-IMMS as a tool for label-free high-throughput screening of variants for libraries of substrates in the same timeframe as single-substrate transformations.

The DiBT-IMMS method demonstrated above for PAL and $\mathrm{P} 450$ reactions can in principle be applied to a broad range of overproduced intracellular enzymes that can be used as whole cell biocatalysts, a rather common condition in the fields of protein engineering and synthetic biology. Concerning the substrate/product scope of our technique, the ability to cross the membranes in order to react and be excreted is a key requirement, along with low volatility and reasonably high ionization efficiency for DESI detection. However, in spite of those limitations, a very broad range of substrates/products can be effectively and rapidly analysed by DiBT-IMMS, e.g., free amines, carboxylic acids, alkaloids, phenols, and so on. Such substrate scope is definitely broader and more flexible than the traditional colorimetric or tag-based screening strategies. ${ }^{1,2}$

In summary, we have shown for the first time that DiBT-IMMS analysis of bacterial colonies from agar plates can be used for the identification of recombinant biocatalysts. Several features of this analytical method make it particularly useful for application in biocatalysis: (i) ambient and direct sampling conditions dramatically reduce liquid handling of samples and allow for in situ extraction of DNA; (ii) activity measurements are label free, thus allowing for universal workflows matching the need to screen many different biocatalytic reactions and libraries of potential substrates; (iii) high resolution of activity measurements by mass allows for multiplexing biocatalytic reactions to match the need for combinatorial sampling (substrate vs. variant libraries); (iv) ambient sampling means that biotransformations can be monitored in real time in live colonies. The potential capabilities of this semi in vivo analytical method enable the directed evolution library screening by integrating the DNA information with analysis results. Therefore, we envisage that this imaging method could find wide application in all areas that require the analysis of biotransformations in recombinant microbial populations, particularly in biotechnology and synthetic biology. 


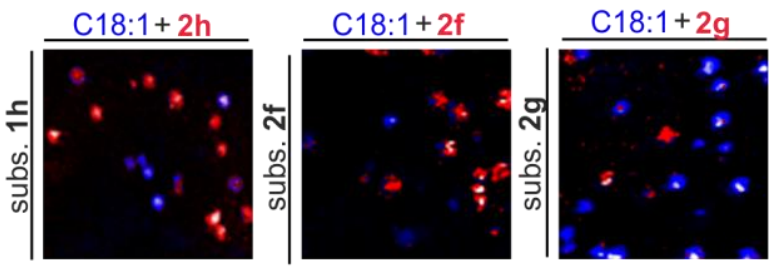

b

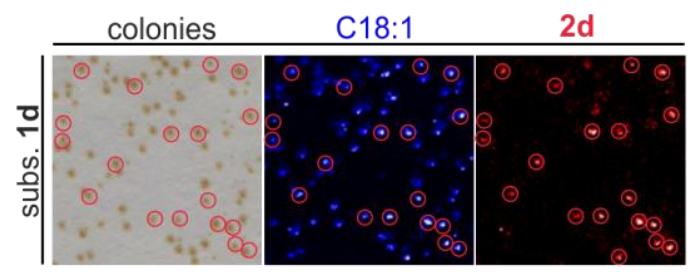

C

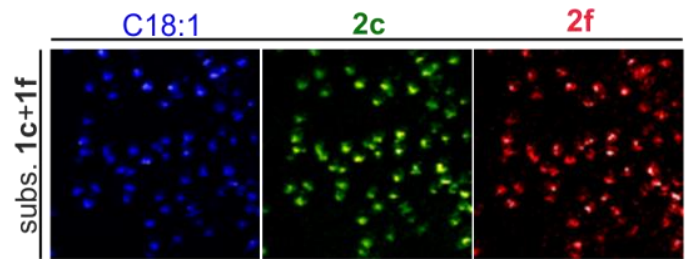

Figure 4. Application of DiBT-IMMS imaging to screen biotransformation reactions with different colonies and/or different substrates. (a) DiBT-IMMS imaging of the PAL reaction with different substrates, performed on mixtures of colonies either producing AvPAL or harbouring an empty vector, in different ratios (blue: C18:1, red: product). (b) DESI-IM-MS imaging of the PAL reaction with 1d, performed on colonies either producing AvPAL or harbouring an empty vector (blue: C18:1, red: product $2 \mathbf{d}$ ), compared with the picture of the original membrane. (c) DiBT-IMMS imaging of the PAL reaction with a mixture of $\mathbf{1 c}$ and $\mathbf{1 f}$, performed on colonies producing AvPAL (blue: C18:1, green: product $\mathbf{2 c}$, red: product $\mathbf{2 f}$ ).

\section{ASSOCIATED CONTENT}

Supporting Information

Experimental section and supplementary figures. This material is available free of charge via the Internet at http://pubs.acs.org.

\section{AUTHOR INFORMATION}

Corresponding Authors

*perdita.barran@manchester.ac.uk

*sabine.flitsch@manchester.ac.uk

ORCID

Cunyu Yan: 0000-0002-3603-2421

Fabio Parmeggiani: 0000-0001-5861-9269

Nicholas J. Turner: 0000-0002-8708-0781

Sabine L. Flitsch: 0000-0003-3974-646X

Perdita E. Barran: 0000-0002-7720-586X

\section{Notes}

The authors declare no competing financial interests.

\section{ACKNOWLEDGMENT}

This work was funded by the Biotechnology and Biological Sciences Research Council (BBSRC) and GlaxoSmithKline (GSK) by grants BB/K00199X/1, BB/L015048/1 and BB/M017702/1. All MS work was performed within the Michael Barber Centre for Collaborative Mass Spectrometry. N.J.T. and S.L.F. are each grateful to the Royal Society for a Wolfson Research Merit Award.

\section{REFERENCES}

(1) (a) Tracewell, C. A.; Arnold, F. H. Curr. Opin. Chem. Biol. 2009, 13, 3-9. (b) Packer, M. S.; Liu, D. R. Nat. Reviews 2015, 16, 379-394. (c) Davids, T.; Schmidt, M.; Böttcher, D.; Bornscheuer, U. T. Curr. Opin. Chem. Biol. 2013, 17, 215-220.

(2) (a) Reymond, J.-L.; Babiak, P., Adv. Biochem. Engin. Biotechnol. 2007, 105, 31-58. (b) Alexeeva, M.; Enright, A.; Dawson, M. J.; Mahmoudian, M.; Turner, N. J., Angew. Chem. Int. Ed. 2002, 41, 3177-3180 (c) Parmeggiani, F.; Lovelock, S. L.; Weise, N. J.; Ahmed, S. T.; Turner, N. J. Angew. Chem. Int. Ed. 2015, 54, 4608-4611.

(3) McLafferty, F. W. Science 1981, 214, 280-287.

(4) (a) Zea, C. J.; MacDonell, S. W.; Pohl, N. L.; J. Am. Chem. Soc. 2003, 125, 13666-13667; (b) Yu, Y.; Ko, K.; Zea, C.J.; Pohl, N. L.; Org. Lett. 2004, 6, 2031-2033; (c) Shen, Z.; Go, E. P.; Gamez, A.; Apon, J. F.; Fokin, V.; Greig, M.; Ventura, M.; Crowell, J. E.; Paulson, J. C.; Stevens, R. C.; Finn, M. G.; Siuzdak, G. ChemBioChem 2004, 5, 921927.

(5) (a) Reetz, M. T.; Becker, M. H.; Klein, H.-W.; Stockigt D.; Angew. Chem. Int. Ed. 1999, 38, 1758-1761. (b) Guo, J.; Wu, J.; Siuzdak G.; Finn, M. G.; Angew. Chem. Int. Ed. 1999, 38, 1755-1758; (c) DeSantis, G.; Wong, K.; Farwell B.; Chatman, K.; Zhu, Z.; Tomlinson, G., Huang, H.; Tan, X.; Bibbs L.; Chen, P.; Kretz, K.; Burk, M. J.; J. Am. Chem. Soc. 2003, 125, 11476-11477.

(6) (a) Takáts, Z.; Wiseman, J. M.; Gologan, B.; Cooks, G. R. Science 2004, 306, 471-473. (b) Cody, R. B.; Laramée, J. A.; Durst, H. D. Anal. Chem. 2005, 77, 2297-2302.

(7) (a) Cooks, R. G.; Ouyang, Z.; Takats, Z.; Wiseman, J. M. Science, 2006, 311, 1566-1570. (b) Harris, G. A.; Galhena, A. S.; Fernandez, F. M. Anal. Chem. 2011, 83, 4508-4538.

(8) (a) Song, Y.; Talaty, N.; Tao, W. A.; Pan, Z.; Cooks, R. G. Chem. Commun. 2007, 61-63. (b) Meetani, M. A.; Shin, Y.-S.; Zhang, S.; Mayer, R.; Basile F. J. Mass. Spectrom. 2007, 42, 1186-1193. (c) Song, Y.; Talaty, N.; Datsenko, K.; Wanner, B. L.; Cooks, R. G. Analyst 2009, 134, 838-841. (d) Wiseman, J. M.; Puolitaival, S. M.; Takats, Z.; Cooks, R. G.; Caprioli, R. M. Angew. Chem. Int. Ed. 2005, 44, 7094-7097.

(9) (b) Wu, C., Dill, A. L., Eberlin, L. S., Cooks, R. G., Ifa, D. R., Mass Spectrom. Rev. 2013, 32, 218-243.

(10) (a) Cornett, D.S.; Reyzer, M. L.; Chaurand P.; Caprioli, R. M.; Nat Methods 2007, 4, 828-833. (b) Laskin, J.; Lanekoff, I. Anal. Chem. 2016, 88, 52-73. (c) Norris, J. L.; Caprioli, R. M. Chem. Rev. 2013, $113,2309-2342$.

(11) (a) Chungtai, K.; Heeren, R. M. A. Chem. Rev. 2010, 110, 3237 3277. (b) Schwamborn, K.; Caprioli, R. M. Nat. Rev. Cancer 2010, 10, 639-646. (c) Spengler B. Anal. Chem. 2015, 87, 64-82.

(12) Watrous, J. D.; Dorrestein, P. C. Nat. Rev. Microbiol. 2011, 9, $683-$ 694.

(13) (a) Borsdorf, H.; Eiceman, G. A. Appl. Spectrosc. Rev. 2006, 41, 323 375. (b) Yan, C.; Schmidberger, J. W.; Parmeggiani, F.; Hussain, S. A.; Turner, N. J.; Flitsch, S. L.; Barran, P. Analyst 2016, 141, 23512355.

(14) Turner, N. J. Curr. Opin. Chem. Biol. 2011, 15, 234-240.

(15) Moffitt, M. M.; Louie, G. V.; Bowman, M. E.; Pence, J.; Noel, J. P.; Moore, B. S. Biochemistry 2007, 46, 1004-1012.

(16) Urlacher, V. B.; Girhard, M. Trends Biotechnol. 2012, 30, 26-36.

(17) O’Reilly, E.; Köhler, V.; Flitsch, S. L.; Turner, N. J. Chem. Commun. 2011, 47, 2490-2501.

(18) Julsing, M. K.; Cornelissen, S.; Bühler, B.; Schmid, A. Curr. Opin. Chem. Biol. 2008, 12, 177-186.

(19) den Braver, M. W.; den Braver-Sewradj, S. P.; Vermeulen, N. P.; Commandeur, J. N. Toxicol. Lett. 2016, 253, 46-54.

(20) O’Reilly, E.; Corbett, M.; Hussain, S.; Kelly, P. P.; Richardson, D.; Flitsch, S. L.; Turner, N. J. Catal. Sci. Technol. 2013, 3, 1490-1492. 
TOC GRAPHIC

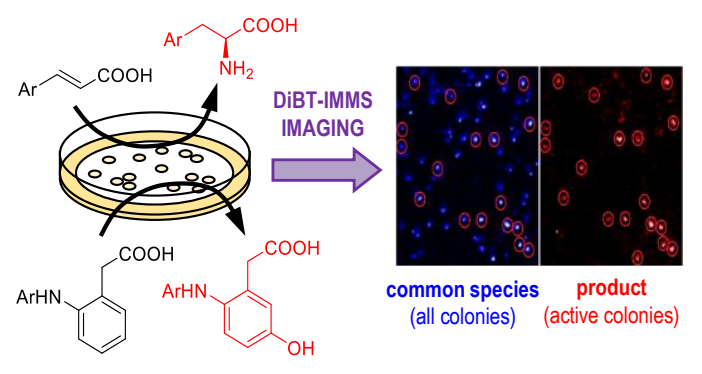

